\title{
Nocardia dassonvillei
}

National Cancer Institute

\section{Source}

National Cancer Institute. Nocardia dassonvillei. NCI Thesaurus. Code C86614.

A species of aerobic, Gram-positive, rod shaped bacteria assigned to the phylum Actinobacteria. This species is spore forming, nonacid-fast, urease negative, catalase positive, hydrolyzes starch, gelatin, esculin, xanthine, and hypoxanthine, reduces nitrate, and produces an aerial hyphae and substrate mycelium that fragments into coccoid elements. N. dassonvillei is found in the environment including mildewed grain and in is pathogenic in humans, though cases are rare. 